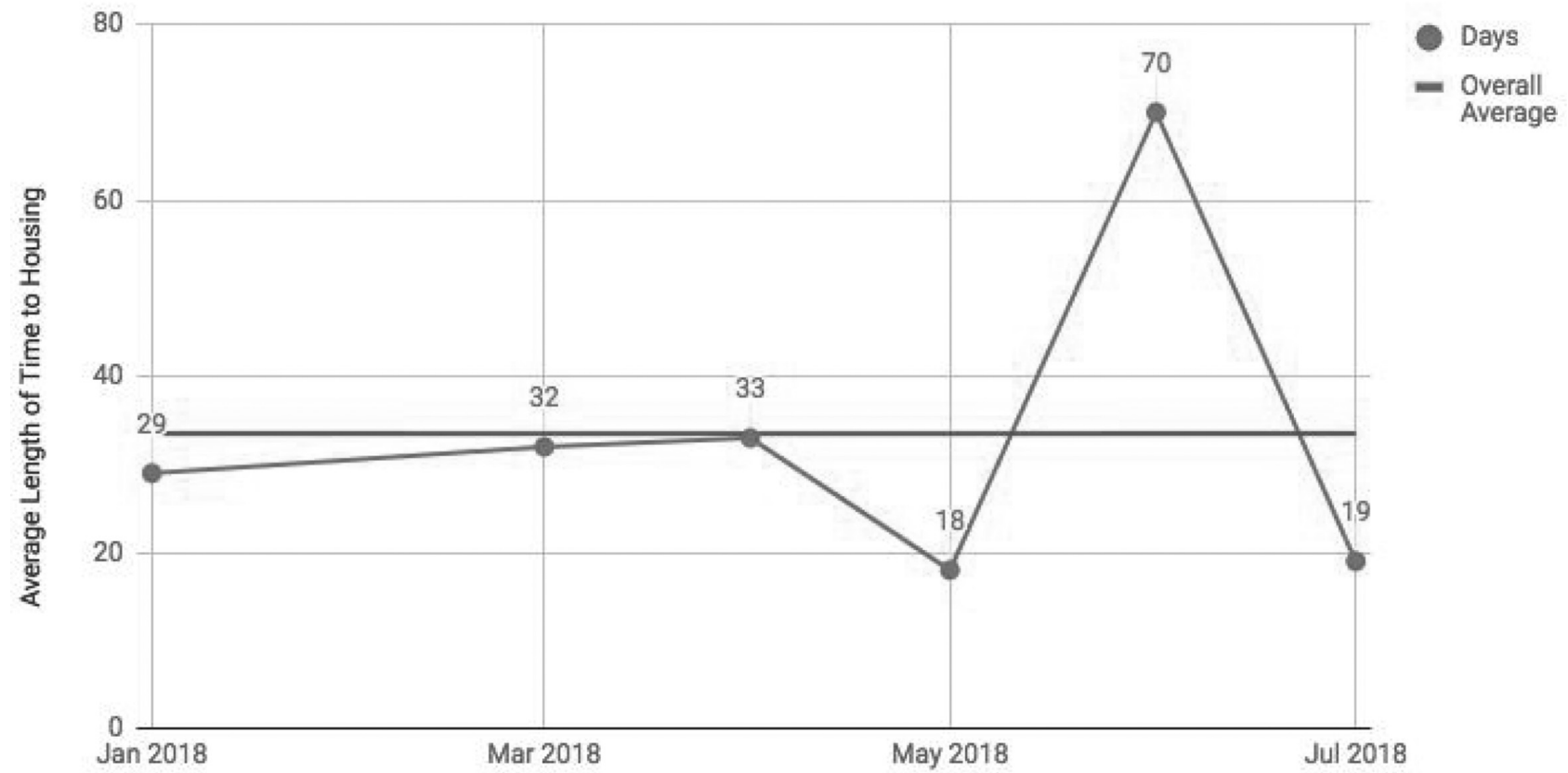

Number of Days

Abstract IHI ID 02 Figure 6 Average length of time from homeless identification to housing placement

\title{
IHI ID 03 EQUIP, AN EVIDENCE-BASED QUALITY IMPROVEMENT PROCESS: IMPROVING THE SPEED TO INSIGHT
}

Jose Benuzillo, Lucy Savitz. Kaiser Permanente, USA

\subsection{6/ihisciabs.3}

EQuIP, An Evidence-based Quality Improvement Process: Improving the Speed to Insight

\section{Literature monitoring in target clinical areas of importance for new comparative effectiveness evidence \\ - Comparative Effectiveness Studies \\ - Systematic Reviews \\ - Other Filtered Scientific Evidence}

Horizon scanning: structured approach that provides a systematic process for exploring dato

Provide parameters for guiding data exploration. Use prior available knowledge to define the scope and to add specificity to the data exploration.

Set the data exploration goals and build a logic model to describe the inputs and the outputs.
Assembling the needed data

from various sources to examine local practices

- EHR Data

- Clinical Databases

- Digital Data

- Claims Data

- Geospatial Data

- Patient-Reported Outcome Measures

Build a robust datoset by combining relevant data sources

Extract only the data relevant for the data exploration.

Spend time cleaning, merging, defining, and validating the data.

This should be a combined effort of

the data scientist and business or

clinical expert.

Try to fit the logic model with the data.
Background Despite the recognition that data can create value for healthcare organizations, only a few have adopted rigorous analytic approaches to support their data exploration efforts. With new sources and increasing amounts of data available to guide quality improvement work, having an evidence-based approach for directing data analytics is essential.
Data analytics

- Traditional Descriptive Statistics

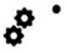

- Traditional QI Tools Root Cause Analysis Pareto Chart
SPC Charts Process Map

- Advanced Analytics

- Geospatial Analysis

Combine different tools to draw insight from your data

Identify patterns, anomalies, and opportunities. Seek to answer the following questions: Who?, When?, Where?, What?, How?, and Why?

Begin with broad descriptions and then focus on areas of value, such as unwarranted variation or worse than expected clinical outcomes. Risk

adjust and use benchmarks for meaningful comparisons.

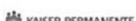
Center for Health Research 
Finding Variation among PCl, Pacemaker, ICD, TAVR and Open Heart Surgery Cases

Leakage

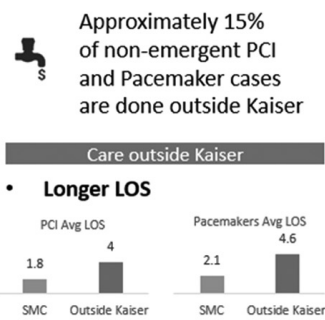

- More Imaging (\$ \& \$

- More expensive

- Higher \% of cases coded with MCC

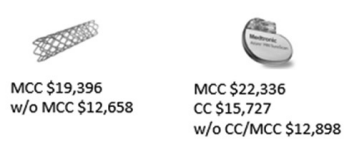

Avoidable LOS

The proportion of same$\because$ day discharged patients was low in general

- $\mathrm{PCI}$

$26 \%$ of patients go home the same day

- Pacemaker Implants $24 \%$ of patients go home the same day

$74 \%$ go home the next day

- ICD Generator Procedures $6 \%$ of patients go home the same day $50 \%$ go home the next day

\section{Potential SDD by Procedure}

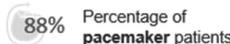

$79 \%$ Percentage of ICD patients

\begin{tabular}{l|lll}
2017 & 590 & 364 & 123 \\
Volume & PCI Procedures & Pacemaker Implants & ICD Generator Procedures \\
\hline
\end{tabular}

Pacemaker Implants

ICD Generator Procedures

30-Day Readmissions

867 open heart surgery cases performed at SMC between 2016-2017

30 day readmission rate $9.9 \%$ - Average cost of CABG readmission $\$ 14,600^{1}$

2017 Readmission Rate

- Extended Care $=\mathbf{1 5 . 4 \%}$

- Home $=8.2 \%$

$2 \mathrm{X}$ more likely to be readmitted if female $>=70$ years old

$3 \mathrm{Y}$ more likely to be

3 readmitted if the patient is female, seventy, and discharged to extended care

456

Open Hearts
Outpatient Shift

Approximately $80 \%$ of $\mathrm{PCI}$

procedures had a hospital status of "Inpatient"

- National benchmark $\mathbf{5 3 \%}$

Quality and Patient Safety

- Appropriate use criteria for procedures performed outside Kaiser

\section{Work in Progress}

- Extending the readmission analysis to HIP \& Knee replacements

- Optimization of TAVR program

- Monitored anesthesia

- Percutaneous femoral access

- No ICU post procedure

\section{Abstract IHI ID 03 Figure 2}

Objectives To propose an evidence-guided systematic approach for exploring data to identify QI opportunities.

Methods We developed a linear framework that begins with monitoring the literature in target clinical areas of importance. This review of existing knowledge by a multidisciplinary team provides parameters that guide data exploration and facilitates the selection of benchmarks and potential balance measures. Then, a robust dataset is built by combining relevant data sources. Next, data are analyzed using a combination of traditional statistical and quality improvement techniques. Lastly, key points are summarized and presented to stake holders as specific improvement opportunities in the context of business value; listing actionable targets with estimated impact.

Results The proposed methodology was tested on one of Kaisers target clinical areas of importance, interventional cardiology. Three potential improvement opportunities were identified:

1. network leakage;

2. avoidable hospital days; and

3. preventable 30 day hospital readmission.

Preliminary estimates suggested that our data could lead to approximately 1 million dollars in savings and up to 250 avoided hospital days while improving the quality and safety of care to our members.
Conclusions Systematic use of a framework for data exploration may create operational and strategic business value by increasing the speed at which data are transformed into actionable knowledge.

\section{IHI ID 04 APPLICATION OF ELECTRONIC TRIGGER TOOL METHODS TO IDENTIFY TARGETS FOR IMPROVING DIAGNOSTIC SAFETY}

${ }^{1}$ Hardeep Singh, ${ }^{1}$ Ashley ND Meyer, ${ }^{2}$ Dean F Sittig, ${ }^{1}$ Daniel R Murphy. ${ }^{1}$ Center for Innovations in Quality, Effectiveness and Safety, Michael E. DeBakey VA Medical Center, and Baylor College of Medicine, USA; ${ }^{2}$ Center for Healthcare Quality and Safety, School of Biomedical Informatics, University of Texas Health Science Center, USA

\subsection{6/ihisciabs.4}

Background Failure to follow-up abnormal test results can lead to patient harm.

Objectives We created and validated electronic trigger algorithms that analyzed electronic health record (EHR) data from a large Veterans Affairs (VA) network to identify patients with potential delays in diagnostic evaluation for multiple cancers.

\begin{tabular}{|c|c|c|c|c|c|c|c|c|}
\hline Cancer Trigger & $\begin{array}{l}\text { Unique Patients } \\
\text { with Trigger } \\
\text { Positives }\end{array}$ & $\begin{array}{l}\text { Unique Patients } \\
\text { Seen }\end{array}$ & Timeframe & PPV $\%(95 \%$ Cl) & NPV $\%(95 \% \mathrm{Cl})$ & $\begin{array}{l}\text { Extrapolated } \\
\text { Sensitivity \% } \\
(95 \% \mathrm{Cl})\end{array}$ & $\begin{array}{l}\text { Extrapolated } \\
\text { Specificity \% } \\
(95 \% \mathrm{Cl})\end{array}$ & $\begin{array}{l}\text { Estimated Number of } \\
\text { Diagnostic Delays } \\
\text { Found per Year }\end{array}$ \\
\hline Bladder & 495 & 310,331 & Jan 2012-Dec 2014 & $58.0(53.0-62.9)$ & $97.0(90.8-99.2)$ & $64.1(59.4-68.5)$ & $96.2(95.6-96.6)$ & 95.7 \\
\hline Breast & 552 & 365,686 & Jan 2010-May 2015 & $70.8(66.0-75.1)$ & $93.0(85.6-96.9)$ & $76.8(72.7-80.4)$ & $90.8(89.2-92.1)$ & 72.2 \\
\hline Colorectal & 1,073 & 245,158 & Jan 2013-Dec 2013 & $56.0(51.0-61.0)$ & $88.0(79.6-93.4)$ & $68.6(65.4-71.6)$ & $81.1(79.5-82.6)$ & 600.9 \\
\hline Hepatocellular & 130 & 333,828 & Jan 2011-Dec 2014 & $82.3(74.4-88.2)$ & $98.0(92.3-99.7)$ & $89.1(81.8-93.8)$ & $96.5(94.8-97.7)$ & 26.7 \\
\hline Lung & 655 & 208,633 & Jan 2012-Dec 2012 & $60.5(55.5-65.3)$ & $97.0(90.8-99.2)$ & $91.7(88.6-94.1)$ & $81.7(79.6-83.7)$ & 396.3 \\
\hline
\end{tabular}

${ }^{*} \mathrm{Cl}=$ Confidence Interval 